\title{
Kernos
}

Revue internationale et pluridisciplinaire de religion grecque antique

19 | 2006

Varia

\section{Généalogies et structures de parenté dans la mythologie grecque}

\section{Evanghelos A. Moutsopoulos}

\section{(2) OpenEdition \\ Journals}

Édition électronique

URL : https://journals.openedition.org/kernos/425

DOI : $10.4000 /$ kernos.425

ISSN : 2034-7871

\section{Éditeur}

Centre international d'étude de la religion grecque antique

\section{Édition imprimée}

Date de publication : 1 janvier 2006

Pagination : 31-34

ISSN : 0776-3824

\section{Référence électronique}

Evanghelos A. Moutsopoulos, « Généalogies et structures de parenté dans la mythologie grecque », Kernos [En ligne], 19 | 2006, mis en ligne le 20 mars 2011, consulté le 24 août 2022. URL : http:// journals.openedition.org/kernos/425 ; DOI : https://doi.org/10.4000/kernos.425 


\section{Généalogies et structures de parenté dans la mythologie grecque}

\footnotetext{
Résumé : Dans la mythologie grecque, les listes généalogiques concourent à la rationalisation de l'univers par le recours aux principes d'identité et de causalité tels qu'ils sont vécus par la mentalité archaïque, chez l'homme désireux de pénétrer le mystère du monde qui l'entoure et dans lequel il ressent le besoin de s'intégrer pour assurer sa survie, avant de tenter de le soumettre à sa volonté par des pratiques magico-religieuses. Ce faisant, l'homme grec, comme tout homme primitif, recourt à une humanisation de l'univers à son image propre, afin de mieux se l'expliquer et de mieux agir sur lui. Cette représentation du monde, qui établit des parallélismes constants, se perpétuera dans la pensée grecque jusqu'à une époque relativement tardive.
}

Abstract: Genealogies and Kinship Structures in Greek Mythology. Genealogical lists in Greek mythology converge towards rationalising the universe by means of the principles of identity and causality as experienced by archaic mentality and primitive man. He feels eager to penetrate into the mystery of the world that surrounds him and into which he feels the need to integrate himself, in order to assure his survival prior to his attempt to subdue it to his own will by means of magical and religious practices. In so doing, the Greek citizen, just like any primitive human being, resorts to the humanisation of the universe through his own image, in order better to interpret it and more accurately to act upon it. Such an image of the world, establishing constant parallel schemata, was to last up to a relatively late era.

Il est impensable de faire état de quelque généalogie en général, et dans la mythologie grecque en particulier, sans tenir compte des structures de parenté et sans se référer, du point de vue méthodologique, à des catégories susceptibles de faciliter le classement, mais aussi la compréhension des liens existant entre dieux et héros, ainsi que l'herméneutique de la nature et du caractère des personnages mythiques impliqués dans une action ${ }^{2}$. Homère et Hésiode, ainsi que les Tragiques qu'ils inspirèrent ${ }^{3}$, fournissent une matière première abondante à cet effet. Les lois cosmiques sont censées expliquer le fonctionnement d'un univers conçu par la mentalité grecque, une mentalité à plusieurs égards encore archaïque, même à l'époque classique, et qui n'est pas encore libérée de l'emprise d'une conception plus ou moins animiste du monde dont les humains dépendent, et dans lequel ils tentent de s'intégrer

\footnotetext{
1 Cf. J. Cazeneuve, La mentalité archaïque, Paris, A. Colin, 1961; cf. Cl. Lévi-STrauss, La pensée sauvage, Paris, Plon, 1962.

${ }^{2}$ Cf. Cl. LÉvi-STRauss, Les structures élémentaires de la parenté, Paris, Plon, 1949; cf. id., Mythologiques, t. 1, Le cru et le cuit, Paris, Plon, 1964, Introduction, p. 9-10.

${ }^{3}$ Cf. E. Moutsopoulos, La transstructuration du mythe dans la tragédie, L'béritage du monde grec (éd. par L. Couloubaritsis), Paris / Bruxelles, Vrin / Ousia, 1989 (Cahiers de philosophie ancienne, 7), p. 31-44; id., Philosophes de l'Égée, Athènes, Fondation de l'Égée, 1991, p. 16-39.
} 
pour le dominer par des voies appropriées et en tenant compte des principes tout puissants d'identité et de causalité . $^{-}$.

D'une manière globale, il est évident que, pour l'esprit dominant de la religion grecque, non seulement l'univers, mais aussi les sociétés divines et humaines fonctionnent selon les lois rigoureuses auxquelles même les dieux ne sauraient échapper; des lois qui toutefois sont loin de n'être pas contradictoires entre elles, sans que cela empêche en quoi que ce soit leur coexistence, conformément à l'expression primitive du principe d'identité. Ces lois sont personnifiées, telles par exemple, celles que représentent Dikè et Heimarménè : Dikè agit d'après la formule qui met en relation l'bybris et la tisis, formule aux termes de laquelle la deuxième se révèle immanquablement comme la conséquence de la première; de son côté, Heimarménè forme la trame de la vie, une trame par rapport à laquelle aucune déviation n'est concevable, alors que toute tentative d'y échapper se voit sévèrement punie, le destin étant $\dot{\alpha} \delta \dot{n} \varrho \iota t o c$, incombattable ${ }^{6}$.

Dans ces conditions, nature et surnaturel s'interpénètrent au niveau du merveilleux et la mythologie, en tant que cadre d'une conception distinctive du monde, s'avère le miroir de l'organisation d'une société : pour ne point perdre son pouvoir, Cronos dévore ses fils, à l'image des souverains primitifs et des animaux dont les mâles dévorent ou étranglent leur progéniture. À défaut d'intervention du facteur merveilleux, c'est la ruse féminine qui intervient alors, par exemple, pour sauver les situations et confirmer l'empire de la nécessité. Aucun subterfuge, même dû à une initiative divine, ne pourra écarter l'inévitable : la parenté naturelle, même divine, et renforcée par une «parenté spirituelle» encore plus puissante, ne suffit pas à neutraliser la vigueur de la destinée humaine qu'est la mort : Thétis confiera Achille, son fils, à Athéna qui croira l'avoir rendu immortel en le soumettant à l'épreuve du feu, mais omettra d'en «immuniser» le talon. Une omission ou une erreur quelconque se trouve au ${ }^{7}$ départ de toute tentative avortée, ne serait-ce que magique, de contourner le sort, en défiant le caractère inéluctable. C'est relativement sur le tard que Platon soutiendra que les affaires des hommes sont régies par la divinité, certes, mais aussi par le hasard et le kairos, le maniement de ce dernier incombant exclusivement à la conscience et à l'action humaines'.

Qui dit parenté entend relation naturelle entre personnes. Or une personne est en principe un être individuel, mais qui peut également constituer, avec d'autres personnes, un être collectif. Souvent ces êtres sont facilement

${ }^{4}$ Cf. L. LÉvY-BruHL, La mentalité primitive, Paris, Alcan, 1922.

Cf. id., Le surnaturel et la nature (1931), nouv. éd., Paris, P.U.F., 1963.

${ }^{6}$ Cf. Iliade XIV, 42; Eschyle, Prométhée, 105; POLYBe, I, 2, 3.

${ }^{7}$ Cf. E. Moutsopoulos, La pensée et l'erreur, Athènes, 1961, p. 53-76.

${ }^{8}$ Sur l'imparable, $c f$. V. Jankelevitch, L'irréversible et la nostalgie, Paris, Flammarion, 1974.

9 Cf. E. Moutsopoulos, «Hasard, nécessité et kairos dans la philosophie de Platon », in Hasard et nécessité dans la philosophie ancienne, Athènes, Académie d'Athènes, 2005, p. 59-69. Cf. cependant Protagoras, 345d; Rép. V, 464e; VI, 489d; Lois VII, 818b. 
discernables : le chœur des Muses est composé de divinités dont chacune possède un caractère distinct et des attributions propres. Il en est de même des «enfants de la Nuit». Il existe cependant des êtres collectifs dont les éléments constitutifs demeurent indissociables dans la mesure où ils ne sont pas nommés expressément : les Titans, à part Prométhée et Épiméthée qui se

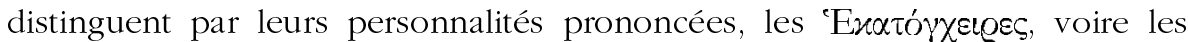
Danaïdes illustrées par Eschyle dans les Suppliantes ${ }^{11}$. Dans ce cas, leurs êtres constitutifs sont tous condamnés à subir le même sort; or il n'est pas improbable qu'à un moment donné d'importance kairique, l'un d'eux se détache du groupe soit pour fuir une destinée qui s'annonce néfaste, soit par esprit d'aventure, pour tenter sa chance ou afin de poursuivre un but précis, ce qui lui vaut alors ou bien de périr ou bien (mais tout à fait exceptionnellement) de gagner finalement son pari. Des deux options, la deuxième est, il est vrai, teintée de romanesque avant la lettre. La règle favorise toutefois la première. Un pessimisme rarement latent, plus souvent prononcé, traverse l'ensemble de la mythologie grecque en l'assombrissant. Des divinités comme Prométhée éclairent évidemment ce paysage d'un rayon passager, mais sont bientôt sanctionnées pour avoir manqué de se soumettre aux lois universelles.

Les structures élémentaires de la parenté sont réductibles à trois catégories qui correspondent à trois types de relations: descendantes, ascendantes, collatérales. Telles qu'elles apparaissent systématiquement chez Hésiode, les généalogies se présentent principalement sous les deux premiers types: mention est faite des parents, puis de la progéniture qui, à l'occasion, comporte plusieurs noms, ce qui renvoie en passant à la catégorie de relations collatérales. En revanche, bien que moins fréquemment, les mêmes relations sont mentionnées selon les cas, en sens inverse ${ }^{12}$. Or il s'agit presque toujours de mentions exceptionnelles, sauf au tout début de la Théogonie: Ciel et Terre y figurent comme des principes divins d'où les autres divinités tirent leur origine ${ }^{13}$. Le recours le plus fréquent au type de relations collatérales concerne la citation des noms de frères et sœurs dont les fonctions et attributions à l'intérieur de l'univers mythique varient par la suite. De variations, la généalogie mythologique en regorge, d'ailleurs : dédoublements et substitutions y alternent sans cesse, preuve de l'existence de diverses sources d'inspiration des poètes qui y recourent. La raison en est, d'une part, qu'elles satisfont le besoin ultérieur d'introduire, dans le système, des fonctions qui, autrement, demeureraient sans répondant; et, d'autre part, de parer à la fusion de cultes de divinités totémiques entièrement différentes à l'origine.

\footnotetext{
${ }^{10}$ Cf., par ailleurs, Cl. RAMnOux, La Nuit et les enfants de la Nuit dans la tradition grecque, Paris, Flammarion, 1959.

${ }^{11}$ Cf. E. Moutsopoulos, « L'être associé », Revue Philosophique 95 (1971), p. 179-182.

12 Par une curieuse coïncidence, on rencontre les mêmes procédés de mentions généalogiques: descendant et ascendant, dans le Nouveau Testament, respectivement au début des Évangiles de Mathieu et de Marc.

${ }^{13}$ On notera également, dans la Genèse, la priorité du ciel et de la terre, mais en tant qu'éléments créés.
} 
Dans la mythologie grecque, les listes généalogiques, concourent à la rationalisation de l'univers par le recours aux principes d'identité et de causalité tels qu'ils sont vécus par la mentalité archaïque, chez l'homme désireux de pénétrer le mystère du monde qui l'entoure et dans lequel il ressent le besoin de s'intégrer pour assurer sa survie, avant de tenter de le soumettre à sa volonté par des pratiques magico-religieuses. Ce faisant, l'homme grec, comme tout homme primitif, recourt à une humanisation de l'univers à son image propre, afin de mieux se l'expliquer et de mieux agir sur lui. Cette représentation du monde, qui établit des parallélismes constants, se perpétuera dans la pensée grecque jusqu'à une époque relativement tardive ${ }^{14}$.

Evanghélos MouTsopoulos

${ }^{14}$ Cf. E. Moutsopoulos, «Les cités humaines comme répliques structurales du monde d'après Zénon de Cittium », Diotima 18 (1990), p. 7-14. 